\title{
Theorems on Existence of Measurable Graph and Selector of Multifuction
}

\author{
RAJ KUMAR SONI \\ Dept. of Mathematics \\ Govt. Girls P.G. College, Rewa (M.P.), 486001, India \\ Affiliated to A.P.S. University, Rewa 486003, India \\ Email: rajkumar.68soni@gmail.com \\ DOI: 10.29322/IJSRP.11.11.2021.p11908 \\ http://dx.doi.org/10.29322/IJSRP.11.11.2021.p11908
}

\begin{abstract}
:
In this paper, we have tried to show that the existence of measurable graph of multivalued function based on the concept of Souslin type as well as existence of measurable selector of multivalued function.

KEY WORDS-Graph and Selector of Multifuction, Souslin Type Multifunction.

\section{INTRODUCTION}

The characteristics of multivalued mapping $\Omega: \mathrm{T} \rightarrow \mathrm{X}$ satisfying various possible conditions of measurability have been studied extensively by various authors AUMANN [1], CASTAING [3], JACOBS [6], KURATOWSKI and RYLL-NARDZEWSKI [8], McSHANE \& WARFIELD [11] from 1965 to 1980. The measurability on T, is introduced depending upon its nature. In case when $\mathrm{T}$ is any non empty abstract set, the measurability is developed by considering the collection of subsets of $\mathrm{T}$, satisfying the $\sigma$ algebra. Moreover, if $\mathrm{T}$ is any arbitrary topological space, then the measurable structure on $\mathrm{T}$ is introduced through Borel $\sigma$-algebra of open subsets of $\mathrm{T}$. In addition to this, if $\mathrm{T}$ is special types of topological space like locally compact space, then measurability on $\mathrm{T}$ is considered through Radon-measure $\mu$. The development of multifunction with their measurability based on properties of graph, this topic begins with 1949, after appearance of selection results of VON-NEUMANN [13]. VON-NEUMANN's result seems to have been little known until around 1965 when AUMANN [1] explored them in mathematical economics and in control theory.
\end{abstract}

In 1974, LEESE [10] defined the multifunction of Souslin type and has proved the existence of measurable selector of Souslin type of multifunctions.

Motivated by the work of these authors, we have tried to investigate to show the measurability of graph of such multivalued function as well as existence of measurable selector.

\section{PRELIMINARIES:}

In this section, we have given some necessary definitions and notations as well as some properties of multivalued mapping without proof but oftenly required in our main results.

\subsection{Definition:}


Let $\mathrm{T}$ and $\mathrm{X}$ be any two sets, then a mapping $\Omega: \mathrm{T} \rightarrow \mathrm{X}$ which assigns to each point $\mathrm{t}$ in $\mathrm{T}$, a set $\Omega(\mathrm{t})$ of points in $\mathrm{X}$ is called a multifunction.

A selector for $\Omega$ is a function f from $T$ into $X$ such that $f(t) \in \Omega(t)$ for each $t \in T$.

let $\mathrm{M}$ be the $\sigma$-algebra of subset of $\mathrm{T}$, then multifunction $\Omega$ is said to be measurable if for any closed subset $\mathrm{B}$ of $\mathrm{X}$, the set

$\Omega^{-1}(\mathrm{~B})=\{\mathrm{t} \in \mathrm{T}: \Omega(\mathrm{t}) \cap \mathrm{B} \neq \phi\} \in \mathrm{M}$

Equivalently,

$$
\begin{aligned}
\Omega^{-1}(\mathrm{G}) & =\{\mathrm{t} \in \mathrm{T}: \Omega(\mathrm{t}) \subset \mathrm{G}\} \in \mathrm{M} \\
& =\mathrm{T} \cap\{\mathrm{t} \in \mathrm{T}: \Omega(\mathrm{t}) \subset \mathrm{G}\} \in \mathrm{M}
\end{aligned}
$$

for every open subset G of X.

\subsection{Definition :}

The graph of multifunction is denoted by $\Omega(\Gamma)$ and defined by-

$\Omega(\Gamma)=\{(\mathrm{t}, \mathrm{x}) \in \mathrm{T} \times \mathrm{X}: \mathrm{x} \in \Gamma(\mathrm{t})\}$

If $\Gamma$ is upper semi continuous implies $\Omega(\Gamma)$ is closed.

\subsection{Definition :}

let $\left\{A_{\sigma_{1} \ldots \ldots . . \sigma_{n}}\right\}$ be a complete collection of sets in a given space, indexed by the set of all finite sequences $\sigma_{1}, \ldots \ldots . . \sigma_{n}$ of positive integers. Then the set-

$$
\mathrm{A}=\bigcup_{\sigma} \bigcap_{\mathrm{n}=1}^{\infty} \mathrm{A}_{\sigma_{1}, \ldots \ldots . \sigma_{\mathrm{n}}}
$$

the union being taken over the collection of all infinite sequence $\sigma$ of positive integer; is said to be obtained from the collection $\left\{A_{\sigma_{1}, \ldots \ldots . . \sigma_{n}}\right\}$ by the souslin operation.

If the set $\left\{\mathrm{A}_{\sigma_{1}, \ldots \ldots . . . \sigma_{\mathrm{n}}}\right\}$ belong to a given class $\mathrm{N}$ of sets then $\mathrm{A}$ will be said to belong to the class souslin - $\mathrm{N}$. We shall use the notation-

$$
A=\bigcup_{\sigma} \bigcap_{n=1}^{\infty} A_{\sigma / n}
$$

Where $\sigma / n$ denotes the finite sequence $\sigma_{1}, \ldots \ldots \sigma_{\mathrm{n}}$.

The measurable space $\mathrm{T}$ will be said to admit the Souslin operation if every subset formed in this way from measurable sets is measurable.

\subsection{Definition:}


Let $\mathrm{T}$ be a measurable space and $\mathrm{X}$ a topological space. Then a multifunction $\Omega: \mathrm{T} \rightarrow \mathrm{X}$ is said to be of Souslin type if there exist a Polish space $\mathrm{P}$, a measurable multifunction $\mathrm{F}: \mathrm{T} \rightarrow \mathrm{P}$ with closed values, and a continuous mapping $\phi: \mathrm{P} \rightarrow \mathrm{X}$ such that, for all $\mathrm{t}$, $\Omega(\mathrm{t})=\phi(\mathrm{F}(\mathrm{t}))$

A multifunction of Souslin type is necessarily be measurable.

$\mathrm{R}$ will denote the class of all set $\mathrm{A} \times \mathrm{B}$ where $\mathrm{A}$ is measurable set in $\mathrm{T}$ and $\mathrm{B}$ is closed set in $\mathrm{X}$. We shall need the following Lemma.

\subsection{Lemma:}

Let $\mathrm{X}_{1}$ be a topological space and $\mathrm{K}^{*}$ the class of sets which are closed and compact in $\mathrm{X}_{1}$. Let $\mathrm{Y}$ be a Souslin-K* subspace of $\mathrm{X}_{1}$. Then if $\mathrm{T}$ is a measurable space which admits the Souslin operation, and $\pi_{1}$, is the canonical projection from $\mathrm{T} \times \mathrm{Y}$ into $\mathrm{T}$, then $\pi_{1}(\mathrm{~A})$ is measurable for every Souslin-R set $\mathrm{A}$ in $\mathrm{T} \times \mathrm{Y}$.

\subsection{Lemma:}

Let $\mathrm{X}, \mathrm{Y}$ be topological space and $\psi: \mathrm{X} \rightarrow \mathrm{Y}$ is a continuous mapping. Then if $\mathrm{T}$ is a measurable space and $\Omega: \mathrm{T} \rightarrow \mathrm{X}$ is a multifunction of Souslin type, so is the multifunction $\mathrm{t} \rightarrow \psi(\Omega(\mathrm{t}))$.

\section{Main Results:}

\subsection{Theorem:}

Let $\mathrm{T}$ be a topological space with Borel $\sigma$-algebra $\mathrm{B}(\mathrm{T})$ of open subsets of $\mathrm{T}$, which admits the Souslin operation, $\mathrm{X}$ be Hausdorff topological space, $\Omega: \mathrm{T} \rightarrow \mathrm{X}$ be a multifunction such that there exist a metrizable compact space $\mathrm{P}$ and upper semi continuous multifunction $=\phi(\mathrm{F}(\mathrm{t}))$. Then $\Omega$ has measurable graph.

\section{Proof:}

Since $\mathrm{P}$ is metrizable compact space, so it has a countable base

Therefore $\mathrm{P}$ is a polish space

Moreover, since $\mathrm{F}$ is upper semi continuous multifunction From $\mathrm{T}$ into $\mathrm{P}$, then for every closed set $\mathrm{B} \subset \mathrm{P}$ -

$\mathrm{F}^{-1}(\mathrm{~B})=\{\mathrm{t}: \mathrm{F}(\mathrm{t}) \subset \mathrm{P}-\mathrm{B}\}$ is open

$\Rightarrow \quad \mathrm{F}^{-1}(\mathrm{~B}) \in \mathrm{B}(\mathrm{T})$

$\Rightarrow \quad \mathrm{F}$ is measurable

From (1) and (2), $\Omega$ is a multifunction of Souslin type 
Since $\Omega(t)=\phi(F(t))$ for each $t$

Now we define a family $\left(\mathrm{U}_{\sigma / \mathrm{n}}\right)$ of closed sets of $\mathrm{P}$.

Suppose $\left(\mathrm{U}_{\mathrm{i}}\right)$ be a countable covering of $\mathrm{P}$ by closed sets of diameter $\leq 1 / 2$.

Then for each $U_{i}$, let $\left\{U_{i j}: j=1,2, \ldots ..\right\}$ be a covering of $U i$ by closed sets of diameter $\leq 1 / 4$.

On continuing this process, we get $\mathrm{U}_{\sigma / \mathrm{n}}$ has diameter $\leq \frac{1}{2^{n}}$, then

it remains to prove -

$$
\mathrm{G}(\Omega)=\bigcup_{\sigma} \bigcap_{\mathrm{n}=1}^{\infty}\left(\mathrm{A}_{\sigma / \mathrm{n}} \times \mathrm{B}_{\sigma / \mathrm{n}}\right)
$$

where $A \sigma / \mathrm{n}=\mathrm{F}^{-1}(\mathrm{U} \sigma / \mathrm{n})$ and $\mathrm{B} \sigma / \mathrm{n}=\phi(\mathrm{U} \sigma / \mathrm{n})$

If $(t, x) \in G(\Omega)$, then there exist a point $y$ in P. s.t.

$$
(\mathrm{t}, \mathrm{y}) \in \mathrm{G}(\Omega) \text { and } \mathrm{x}=\phi(\mathrm{y})
$$

Also $\exists$ a sequence $\sigma$ of positive integers s.t. $\{y\}=\bigcap_{n=1}^{\infty} U_{\sigma / n}$

$\Rightarrow \quad \mathrm{F}(\mathrm{t})$ meets $\mathrm{U} \sigma / \mathrm{n}$ for each $\mathrm{n}$ and $\mathrm{x} \in \phi(\mathrm{U} \sigma / \mathrm{n})$ for each $\mathrm{t}$.

$\Rightarrow \quad(t, x) \in A \sigma / n \times B \sigma / n$ for all $n$

$\Rightarrow \quad(\mathrm{t}, \mathrm{x}) \in \mathrm{G}(\Omega)$

$\Rightarrow \quad \Omega$ has measurable graph.

3.2 Theorem: Let $\mathrm{T}$ be a topological space with Borel $\sigma$-algebra and $\mathrm{X}$ be a Hausdorff space then continuous image of metrizable compact space $\mathrm{P}$ and $\Omega: \mathrm{T} \rightarrow \mathrm{X}$ is a compact valued measurable multifunction. Then $\Omega$ has a measurable selector.

Proof: Suppose $\mathrm{X}=\phi(\mathrm{P})$ where $\phi$ is continuous mapping.

Since $\mathrm{P}$ is compact matrizable.

$\Rightarrow \quad \mathrm{P}$ has a countable base $\left(\mathrm{U}_{\mathrm{n}}\right)$ of open sets

Also $\Omega=\Omega_{0}$ be a compact valued measurable multifunction from $\mathrm{T}$ to $\mathrm{X}$.

Now define $F_{n}$ inductive, so that $F_{n}$ is refinement of $F_{n-1}$ by 


$$
\mathrm{B}_{\mathrm{n}}=\phi\left(\mathrm{U}_{\mathrm{n}}\right) \text { for each } \mathrm{n} \geq 1 .
$$

Thus $<\Omega_{\mathrm{n}}>$ is a decreasing sequence of compact valued measurable multifunction.

So, $\quad \mathrm{G}=\bigcap_{\mathrm{n}} \Omega_{\mathrm{n}}$ is measurable.

To prove this theorem, we show that $G(t)$ consists of a single point $f(t)$ (say), for each $t$, then $f$ is the required measurable selector.

Suppose that $\mathrm{y} \in \mathrm{G}(\mathrm{t})$ and $\mathrm{z} \neq \mathrm{y} \exists$ a closed nbd $\mathrm{V}$ of $\mathrm{y}$ not containing $\mathrm{z}$.

$\therefore \quad \mathrm{y}=\phi(\mathrm{p})$ for some $\mathrm{p} \in \mathrm{A}$,

$\exists$ n s.t. $\phi\left(\mathrm{U}_{\mathrm{n}}\right) \subseteq \mathrm{V}$

$\Rightarrow \quad \mathrm{B}_{\mathrm{n}} \subseteq \mathrm{V}$

Now $F_{n-1}(t)$ meet $B_{n}$ in $y$

So that $\mathrm{F}_{\mathrm{n}}(\mathrm{t}) \subseteq \mathrm{B}_{\mathrm{n}} \subseteq \mathrm{V}$

Thus $\mathrm{z} \notin \mathrm{F}_{\mathrm{n}}(\mathrm{t})$

$\Rightarrow \quad \mathrm{z} \notin \mathrm{G}(\mathrm{t})$

$\Rightarrow \quad \mathrm{G}(\mathrm{t})$ consists single point $\mathrm{y}$

$\Rightarrow \quad \Omega$ has measurable selector.

\section{Reference}

1. Aumann R.J., "Integral of set value function". J. Math. Anal. Appl. 12 (1965), 1-12.

2. Bourbaki N., " General Topology part- 2", Addition-Wesley, Reading, Mass., 1966.

3. Castaing C., "Sur Les multifunction measurables", Rev. Francaise Informat. Research operationebel 1 (1967), 91-126.

4. Fillippov A.F., "On certain questions in the theory of optimal control", SIAM J. Control Sec. A 1(1962-63), 76-84.

5. Himmelberg C.J. and Van-Vleck F.S., "Selection and implicit function theorems for multifunction with souslin graph", Bull. Acad. Polon. Sci. Sec. Sci. math. Astronom. Phys. 19 (1971), 911- 916.

6. Jacobs M.Q., "Measurable multivalued mapping and Lusin's theorrem", Trans. Amer. Math. Sco. 134 (1968) pp 471-481.

7. Kuratowski K., "Topology vol. I" New-York- London- Warszawa, (1966).

8. Kuratowski K. and Rvll-Nardzewski C., " A general theorem on selector", Bull. Acad. Polon. Sci. Ser. sci. Math. Astronom. Phys. 13 (1965), 397-403. 
International Journal of Scientific and Research Publications, Volume 11, Issue 11, November 2021

9. Lusin N., "Lecons Sur les Ensembles Analyliques et Leurs Appl. Chelsea, New-York (Second edition) 1972, (First edition was 1930).

10. Leese S.J., "Multifunction of Souslin type", Bull-Austral Math. Soc. 11 (1974) pp. 395-411.

11. McShane E.J. and Warfield R.B., " On Fillippov's implicit function lemma", Proc. Ames. Math. Soc. 18 (1967), $41-47$.

12. Robertson A.P., "On measurable selection", Proc. Roy. Soc. Edinburgh Ser. A 72 (1972-73), 1- 7 (1974).

13. Von-Neumann J., "On rings on operators reduction theory" anal. Math. 50 (1949), pp. 401-485. 